\title{
Welche Risiken und Nebenwirkungen hat Psychotherapie? - Entwicklung des Inventars zur Erfassung Negativer Effekte von Psychotherapie (INEP)
}

\author{
Inga Ladwig ${ }^{a}$ Winfried Rief ${ }^{a} \quad$ Yvonne Nestoriuc $^{b}$ \\ ${ }^{a}$ Klinische Psychologie und Psychotherapie, Universität Marburg, Deutschland \\ ${ }^{\text {b}}$ Fakultät für Erziehungswissenschaft, Psychologie und Bewegungswissenschaft, Universität Hamburg, Deutschland
}

\section{Schlüsselwörter}

Psychotherapieforschung - Therapieergebnisse . Therapeutische Beziehung . Unerwünschte Effekte . Nebenwirkungen - Unethisches Therapeutenverhalten . Therapieschäden

\section{Zusammenfassung}

Hintergrund: Negative Effekte von Psychotherapie sind bis heute wenig systematisch untersucht. Diese Studie stellt die Konstruktion eines Selbstbeurteilungsverfahrens zur Erfassung negativer Effekte von Psychotherapie vor. Patienten und Methoden: Ein Itempool zur breiten Erfassung möglicher erlebter negativer Veränderungen durch Psychotherapie in den Bereichen intrapersonelle Veränderungen, Partnerschaft, Freunde und Familie, Arbeitsplatz, therapeutisches Fehlverhalten und Stigmatisierung wurde über Literaturrecherchen und Expertenbefragungen generiert. Items wurden bipolar formuliert, um ein negatives Priming zu verhindern. Zusätzlich wurde die jeweilige Attribution der Veränderung auf die Psychotherapie oder andere externe Ursachen erfragt. Im Zeitraum von November 2010 bis Februar 2011 nahmen 195 ehemalige Psychotherapiepatienten $(74,9 \%$ weiblich; Alter $\mathrm{M}=38,4$ Jahre; $\mathrm{SD}=11,8)$ an einer Onlineuntersuchung teil, bei der auch die Rahmenbedingungen der Psychotherapie erfragt wurden. Ergebnisse: Von $195 \mathrm{Be}-$ fragten gaben $93,8 \%(n=183)$ an, negative Effekte durch ihre Psychotherapie erlebt zu haben. Die höchsten Raten erlebter negativer Effekte ergaben sich in den Bereichen intrapersonelle Veränderungen $(15,8 \%)$, Stigmatisierung $(14,9 \%)$ und Partnerschaft $(12,0 \%)$ Schwerwiegendes therapeutisches Fehlverhalten wie sexuelle Belästigung $(2,6 \%)$ oder körperliche Gewalt $(1 \%)$ durch den Therapeuten hatten eine geringe Prävalenz. Anhand von Itemanalysen sowie inhaltlichen Kriterien wurde das Inventar zur Erfassung negativer Effekte von Psychotherapie (INEP) mit 21 Items erstellt $(\alpha=0,86)$. Diskussion: Ein Großteil der negativen Veränderungen trat innerhalb des therapeutischen Settings auf (z.B. verletzende Aussagen des Therapeuten, Phasen der Niedergeschlagenheit). Zudem wurden mehr negative Effekte genannt, wenn die therapeutische Beziehung als negativ beschrieben wurde. Schlussfolgerung: Negative Effekte von Psychotherapien sind feststellbar und können mittels Patientenbefragungen und einer systematischen Analyse, via INEP erfasst werden. Die Analyse des Instruments in weiteren klinischen Subpopulationen ist notwendig.

\author{
Keywords \\ Psychotherapy research - Therapy outcome . \\ Therapeutic alliance · Adverse effects . \\ Side effects . Unethical therapist behavior . \\ Negative treatment effects
}

\section{Summary}

What are the Risks and Side Effects to Psychotherapy? Development of an Inventory for the Assessment of Negative Effects of Psychotherapy (INEP)

Background: Potential negative effects of psychotherapeutic treatment have been little studied systematically to date. The current report describes the development of a self-report instrument for assessing negative effects of psychotherapy. Patients and Methods: Items assessing negative effects of psychotherapy in different areas of life (intrapersonal change, relationships, friendships, family, malpractice, and stigmatization) were generated via literature research and presented to a group of psychotherapy experts. Items were created with a bipolar scale to avoid negative priming. Additionally, patients' attributions regarding the cause of negative effects were assessed. Between November 2010 and February 2011, 195 former psychotherapy patients (74.9\% female, age $M=38.4$ years, $S D=11.8$ ) took part in an online survey, also reporting on treatment conditions during therapy. Results: Of 195 participants, $93.8 \%(n=183)$ have reported to have experienced negative effects in or after psychotherapy. The highest rates of negative effects were reported for intrapersonal changes (15.8\%), stigmatization $(14.9 \%)$, and relationships $(12.0 \%)$. Reports of malpractice were few, with $2.6 \%$ sexual harassment, or $1 \%$ physical violence. On the basis of item analysis and content criteria, the Inventory for the Assessment of Negative Effects of Psychotherapy (INEP) comprising 21 items was created (Cronbach's $\alpha=0.86$ ). Discussion: A significant number of negative effects were reported within the therapeutic setting (e.g., feeling offended by what the therapist said; stages of dejection). Additionally, patients who described the therapeutic alliance as poor also reported a high number of negative effects. Conclusion: Negative effects of psychological treatment can be identified and systematically assessed via patient survey and INEP. Further evaluation in different clinical subpopulations is needed.

\section{KARGER \\ Fax +497614520714}

Information@Karger.com

www.karger.com (c) 2014 S. Karger GmbH, Freiburg

1016-6262/14/0244-0252\$39.50/0

Accessible online at:

www.karger.com/ver
Dipl.-Psych. Inga Ladwig

Klinische Psychologie und Psychotherapie

Universität Marburg

Gutenbergstraße 18, 35032 Marburg, Deutschland

inga.ladwig@staff.uni-marburg.de 


\section{Einleitung}

An der Effektivität von Psychotherapie bestehen keine Zweifel. Zahlreiche Studien liefern eindeutige Belege für die erfolgreiche Behandlung unterschiedlicher psychischer Störungen mit den Methoden der gängigen Therapieverfahren [Smith und Glass, 1977; Wampold, 2001]. Wirksamkeitsforschung allerdings bedeutet, wie aus der Medizin bekannt, neben der Untersuchung der Hauptwirkungen auch eine Beschäftigung mit den potenziellen negativen Effekten einer Behandlung; jeder Eingriff in die biochemischen, physikalischen oder eben psychischen Abläufe im Körper eines Menschen wird fast zwangsläufig solche negative Effekte mit sich bringen.

Eine Beschäftigung mit negativen Effekten ist bisher in der Psychotherapieforschung stark vernachlässigt worden. Eine Literaturrecherche deutsch- und englischsprachiger Fachpublikationen im Jahre 2012 ergab nur vereinzelt Treffer und zeigt, dass die Erforschung möglicher negativer Effekte von psychotherapeutischen Interventionen eher unterrepräsentiert ist, gerade im Vergleich zur Wirksamkeitsforschung. Zudem wird deutlich, dass innerhalb der existierenden Studien von verschiedenen Konzepten wie therapeutischem Misserfolg, Therapierisiko, Kosten, Schäden oder Nebenwirkungen gesprochen wird; dabei gibt es bisher keine einheitliche Verwendung dieser Begriffe. Eine Definition der Begrifflichkeiten ist daher von äußerster Relevanz. Wie in der pharmakologischen Wirksamkeitsforschung wäre es auch für die psychotherapeutische Wirksamkeitsforschung selbstverständlich, die Begriffe «Hauptwirkungen» und «Nebenwirkungen» zu verwenden. Nach gesetzlicher Definition erfasst der Begriff Nebenwirkungen jedoch ausschließlich die «beim bestimmungsmäßigen Gebrauch auftretenden schädlichen unbeabsichtigten Reaktionen» (Arzneimittelgesetz (AMG) § 4 Abs. 1, Satz 13). Veränderungen und Schädigungen nach Behandlungsfehlern sind davon ausgeschlossen. Da die Behandlungsqualität in der Psychotherapie jedoch umso schwieriger zu bestimmen ist, wird folglich ein Begriff benötigt, der sowohl Veränderungen und Schädigungen in verschiedenen Lebens- und Funktionsbereichen nach lege artis angewandten Therapien berücksichtigt als auch negative Effekte beschreibt, die aus Behandlungsfehlern des Therapeuten resultieren. Daher schlagen wir den Begriff der negativen Effekte mit folgender Definition vor.

\section{Negative Effekte von Psychotherapie}

Negative Effekte von Psychotherapie werden als Veränderungen definiert, die vom Patienten als negativ erlebt werden und eine direkte oder indirekte Schädigungen darstellen, oder von der betroffenen Person als Nachteil erlebt werden. Diese Veränderung sollte in mindestens einem Funktions- und/oder Lebensbereich des Patienten auftreten sowie zeitlich während der Therapie, unmittelbar danach oder auch erst verzögert. Negative Effekte werden vom Patienten auf die Therapie und nicht auf andere externe Einflüsse zurückgeführt. Unter negative Effekte fällt auch unethisches Fehlverhalten durch den Therapeuten.
Bisherige Studien mit naturalistischen klinischen Stichproben [Lambert und Ogles, 2004; Mohr, 1995] zu negativen Effekten von Psychotherapie berichten Verschlechterungsraten von $5-10 \%$ auf der Symptomebene. Einige wenige Studien berichten negative Effekte auch in anderen Lebensbereichen der Patienten wie Abhängigkeit vom Therapeuten als Lebensberater, ohne den keine eigenständigen Entscheidungen getroffen werden können [Schmoll, 2012], oder auch vermehrt auftretende Probleme in der Partnerschaft nach erfolgreicher Psychotherapie [Hand und Lamontagne, 1976]. Andere negative Effekte sind Hoffnungslosigkeit und Motivationseinbrüche bei ausbleibendem Erfolg und ersten Rückschlägen, Problemaktualisierung oder Angstausweitung, z.B. im Rahmen einer Expositionstherapie, das «Übertrainieren» sozialer Fertigkeiten bis hin zu Egozentrismus, Angst vor Stigmatisierung oder Problemen beim Abschluss von neuen Versicherungen im Anschluss an die Behandlung (für eine ausführliche Diskussion siehe Nestoriuc und Rief [2012]). Dabei können Veränderungen sowohl bei erfolgreicher Therapie hinsichtlich der Hauptsymptomatik als auch bei Ausbleiben des Therapieerfolgs auftreten. Beispielsweise kann ein Patient eine Verbesserung seiner Depressionssymptomatik erleben, dabei jedoch vermehrt in Konflikte mit Familie und Freunden geraten, weil er während der Therapie gelernt hat, eigene Bedürfnisse durchzusetzen, welche er sich zuvor nicht zugestanden hatte. Diese abseits der erfolgreich behandelten Symptomatik entstandenen Probleme können neuen Leidensdruck verursachen. Daraus folgt einerseits eine ethische Pflicht zur Aufklärung der Patienten, um eine autonome Patientenentscheidung für oder gegen die Behandlung zu ermöglichen, und andererseits eine moralisch-praktische Pflicht, negative Effekte zu erkennen, um individuelle Copingstrategien zu finden. Dieser Pflicht kann bisher jedoch nur unzureichend nachgekommen werden, da wissenschaftliche Evidenz darüber fehlt, welche negativen Effekte mit welcher Häufigkeit tatsächlich von den Patienten erlebt werden.

Ziele der vorliegenden Studie sind deshalb die Entwicklung und Evaluation eines Instruments zur systematischen Erfassung negativer Effekte von Psychotherapie und die Analyse von Häufigkeiten negativer Effekte und deren Korrelate auf Patienten- und Therapeutenseite.

\section{Patienten und Methoden}

Itemkonzeption

Über eine Literaturrecherche wurde ein Itempool von 120 möglichen negativen Effekten von Psychotherapie, inklusive verschiedener Aspekte therapeutischen Fehlverhaltens, zusammengestellt. Dieser wurde einer Gruppe von 8 Experten der Psychotherapieforschung in Deutschland vorgelegt und hinsichtlich ihrer Relevanz, Deutlichkeit und Redundanz qualitativ bewertet. Mithilfe dieser Urteile und nach inhaltlichen Überlegungen wurden 52 geeignete Items durch W.R. und Y.N. ausgewählt, die im Rahmen der vorliegenden Internetstudie empirisch evaluiert wurden. Ziel war dabei die Erstellung eines ökonomischen Fragebogens zur Erfassung negativer Effekte von Psychotherapie. Die Items sind nach Mög- 
lichkeit im bipolaren Format 7-stufig formuliert $(-3=$ negative Veränderung trifft völlig zu; $0=$ unverändert; $3=$ positive Veränderung trifft völlig $\mathrm{zu}$ ), um neben Verschlechterungen auch Verbesserungen oder ausbleibende Veränderungen erfassen zu können und ein Bias im Sinne eines negativen Primings zu verhindern [Heuer et al., 2007]. Wenn ein bipolares Format inhaltlich nicht sinnvoll war, wurde ein 4-stufiges unipolares Antwortformat gewählt $(0=$ trifft gar nicht zu; $3=$ trifft völlig zu). $\mathrm{Zu}$ jedem Item wurde die Attribution aus Patientensicht erfasst ( Worauf führen Sie diese Veränderung zurück?» - «auf die Therapie» oder «auf andere Lebensumstände»). Für die Auswertung wurden nur negative Effekte berücksichtigt, die vom Patienten direkt auf die psychotherapeutische Behandlung zurückgeführt wurden.

Zusätzlich zu den 52 Items wurden weitere 15 Fragen ausgewählt, die Erwartungen an die Psychotherapie (jeweils 6 Items), die Qualität der therapeutischen Beziehung (jeweils 6 Items) und den allgemeinen Therapieerfolg erfassen (jeweils 3 Items; Anhang 1; www.karger.com/? DOI=000367928). Das Antwortformat entsprach dem der Fragen zu negativen Effekten.

\section{Datenerhebung und Teilnehmer}

Bei der vorliegenden Studie handelt es sich um eine Onlinebefragung. Die Umfrage wurde mithilfe der Unipark-Software Version 6.1 erstellt. Insgesamt besuchten 586 Personen die Befragungshomepage, und 319 von ihnen stimmten einer Teilnahme zu. Von diesen 319 Teilnehmern beendeten 200 Personen (61\%) die Befragung vollständig. Diese Schwundquote von $39 \%$ entspricht Verlustraten in anderen Internetstudien [Westermann et al., 2012].

Zur Teilnahme an der Erhebung wurden ehemalige Psychotherapiepatienten über Aufrufe auf Internetseiten oder schriftliche Mitgliedsbenachrichtigungen verschiedener eingetragener Vereine zur Hilfe und Selbsthilfe psychisch erkrankter Personen sowie über Aufrufe in sozialen Netzwerken eingeladen:

- Angst-Hilfe e.V. DASH/MASH (www.panik-attacken.de)

- Beratungs- und Informationsserver zu Essstörungen (www.ab-server.de)

- Gegen-Missbrauch e.V. (www.gegen-missbrauch.de)

- Deutsche Gesellschaft Zwangserkrankungen e.V. (www.zwaenge.de)

- Chronic Fatigue Syndrom Forum (www.cfs-treffpunkt.de)

- Angst- und Panikforum (www.angstforum.info)

- Deutsches Bündnis gegen Depressionen e.V.

- Facebook; StudiVZ.

Der schriftliche Aufruf zur Teilnahme war für alle Foren im folgenden Wortlaut gleich formuliert: «Erfahrungen mit Psychotherapie? - Teilnehmer für Umfrage gesucht! Wenn Sie selbst schon Erfahrungen mit Psychotherapie gesammelt haben und interessiert sind, Ihre Erlebnisse mitzuteilen, möchten wir Sie gerne einladen, an unserer Online-Umfrage teilzunehmen».

Ehemalige Psychotherapiepatienten konnten von November 2010 bis Februar 2011 an der Untersuchung teilnehmen. Einschlusskriterien waren ein Lebensalter von mindestens 18 Jahren, ausreichende Deutschkenntnisse sowie eine bereits in Anspruch genommene psychotherapeutische Behandlung. Nach einer Aufklärung über die Studieninhalte und einem eingeholten Einverständnis zur Studienteilnahme wurden den Probanden zunächst 52 Items zu möglichen negativen Effekten ihrer Psychotherapie vorgelegt und ihre Erwartungen gegenüber der Behandlung erfasst. Zudem wurden die Teilnehmer zur Qualität der therapeutischen Beziehung befragt (jeweils 6 Items). Falls bereits mehr als eine Psychotherapie absolviert wurde, sollten die Probanden sich auf die jüngste Behandlung beziehen. Im Anschluss wurden sie zu persönlichen soziodemografischen Merkmalen (Alter, Geschlecht, Nationalität, Familienstand, Bildungsstand und berufliche Situation) und Rahmenbedingungen der zurückliegenden Psychotherapie befragt. Erfasst wurden Therapieform (ambulant/stationär), Therapiedauer, theoretische Ausrichtung und spezifische Interventionselemente, Stundenanzahl, Therapeutengeschlecht, Therapeutenwechsel, früherer Therapieabbruch und frühere Therapieerfahrung sowie Diagnose bei Behandlungsbeginn. Bei den Angaben han-
Tab. 1. Soziodemografische und klinische Merkmale der Stichprobe (Selbstauskunft der Teilnehmer)

\begin{tabular}{|c|c|}
\hline Merkmale & $\mathrm{N}=195$ \\
\hline Geschlecht (weiblich), n (\%) & $146(74,9)$ \\
\hline Alter, $\mathrm{M}(\mathrm{SD})$, range & $38,4(11,8), 18-75$ \\
\hline Allein lebend, n (\%) & $96(49,2)$ \\
\hline \multicolumn{2}{|l|}{ Bildung ${ }^{\mathrm{a}}, \mathrm{n}(\%)$} \\
\hline Kein Abschluss & $2(1)$ \\
\hline Hauptschule & $13(6,7)$ \\
\hline Realschule & $62(32,1)$ \\
\hline Abitur & $56(29)$ \\
\hline Hochschulabschluss & $59(30,6)$ \\
\hline Sonstiger Abschluss & $1(0,5)$ \\
\hline \multicolumn{2}{|l|}{ Berufsstand $^{\mathrm{b}}, \mathrm{n}(\%)$} \\
\hline Angestellt & $89(46,3)$ \\
\hline Selbstständig & $18(9,4)$ \\
\hline Hausfrau/mann & $6(3,1)$ \\
\hline Schüler/Studierender/in Ausbildung & $33(17,2)$ \\
\hline Pensioniert & $29(15,1)$ \\
\hline Erwerbslos & $17(8,9)$ \\
\hline \multicolumn{2}{|l|}{ Psychische Diagnose $^{c}, \mathrm{n}(\%)$} \\
\hline Depression & $121(62,1)$ \\
\hline Angststörung & $103(52,8)$ \\
\hline Persönlichkeitsstörung & $52(26,7)$ \\
\hline Essstörung & $40(20,5)$ \\
\hline Schizophrenie & $4(2,1)$ \\
\hline Sonstige & $10(5,1)$ \\
\hline \multicolumn{2}{|l|}{$\begin{array}{l}\text { M = Mittelwert; SD = Standardabweichung. } \\
{ }^{a} \text { Aufgrund von fehlenden Werten } n=193 \text {. } \\
\text { bैAufgrund von fehlenden Werten } n=192 . \\
{ }^{c} \text { Mehrfachantworten möglich. }\end{array}$} \\
\hline
\end{tabular}

delte es sich um eine Selbstauskunft der Patienten, da keine offiziellen Unterlagen zur Behandlung vorlagen.

Um Verzerrungseffekte bei der Erinnerung der zurückliegenden Psychotherapie zu minimieren, wurde eine Ausreißeranalyse der Variable «Dauer seit Therapieende» durchgeführt. Die Variable wurde standardisiert (Z-transformiert), um einen geeigneten Cutoff zu finden. Gemäß Bortz und Schuster [2010] sind Werte, die mehr als 2 Standardabweichungen vom Mittelwert entfernt liegen, als Ausreißer definiert (Z-Wert $>3,29$ ). Aus diesem Grund wurden 5 Personen aus der Analyse ausgeschlossen, deren Psychotherapie länger als 5255 Tage, d.h. über 14 Jahre zurücklag. In der neu entstandenen Stichprobe $(\mathrm{n}=195)$ lag die Behandlung nun im Mittel 1,99 Jahre zurück (Standardabweichung $(\mathrm{SD})=2,56$ Jahre; Range $=0-12,33$ Jahre). Die nachfolgenden Analysen wurden zunächst für beide Stichproben durchgeführt, um den Impact der Ausreißer festzustellen. Die Ergebnisse unterscheiden sich nur geringfügig, wobei die Stichprobe mit der erweiterten Varianz $(n=200)$ einige wenige Effekte unterdrückte. Aus diesem Grund werden im folgenden Ergebnissteil die Resultate der bereinigten Stichprobe $(\mathrm{n}=195)$ berichtet.

\section{Statistische Auswertung}

Die statistische Auswertung wurde mit SPSS Version 17.0 vorgenommen. Es wurden zunächst deskriptive Analysen durchgeführt und Itemkennwerte berechnet. Anschließend wurde eine Hauptkomponentenanalyse durchgeführt, um die zugrunde liegende Struktur des Fragebogens zu untersuchen. Um erste Hinweise zu möglichen Korrelaten von negativen Effekten von Psychotherapie zu bekommen, wurde im Anschluss eine schrittweise hierarchische Regression berechnet. 
Tab. 2. Charakteristika der Psychotherapie (Selbstauskunft der Teilnehmer)

\begin{tabular}{|c|c|}
\hline Merkmale & $\mathrm{N}=195$ \\
\hline Therapeutengeschlecht (männlich), n (\%) & $65(33,3)$ \\
\hline \multicolumn{2}{|l|}{ Behandlungssetting, $\mathrm{n}(\%)$} \\
\hline Ambulant & $146(74,9)$ \\
\hline Stationär & $49(25,1)$ \\
\hline \multicolumn{2}{|l|}{ Behandlungsmodalität, n (\%) } \\
\hline Einzel & $147(75,4)$ \\
\hline Gruppe & $2(1)$ \\
\hline Einzel + Gruppe & $46(23,6)$ \\
\hline \multicolumn{2}{|l|}{ Therapieumfang in Sitzungen, $\mathrm{n}(\%)$} \\
\hline $1-5$ & $14(7,2)$ \\
\hline $6-25$ & $54(27,7)$ \\
\hline $26-120$ & $85(43,6)$ \\
\hline $121-300$ & $29(14,9)$ \\
\hline$>300$ & $13(6,7)$ \\
\hline \multicolumn{2}{|l|}{ Theoretische Ausrichtung (nach Patientenaussage), n (\%) } \\
\hline VT & $74(37,9)$ \\
\hline PD & $61(31,3)$ \\
\hline GT & $40(20,5)$ \\
\hline Sonstige & $20(10,3)$ \\
\hline Vorzeitiger Therapieabbruch, n (\%) & $43(22,1)$ \\
\hline Frühere Therapieerfahrungen, $\mathrm{n}(\%)$ & $123(63,1)$ \\
\hline Zeit seit Ende der Behandlung in Jahren, M (SD), range & $1,99(2,56), 0-12,33$ \\
\hline $\begin{array}{l}\text { «Insgesamt wurden meine Erwartungen an die Therapie erfüllt / } \\
\text { nicht erfüllt.», M (SD), range }\end{array}$ & $0,3(2,3),-3-3$ \\
\hline $\begin{array}{l}\text { «Ich erlebte die Beziehung zu meinem Therapeuten/ meiner } \\
\text { Therapeutin insgesamt als positiv/negativ.», M (SD), range }\end{array}$ & $1,3(1,8),-3-3$ \\
\hline
\end{tabular}

\section{Ergebnisse}

\section{Stichprobe}

Die Studienteilnehmer umfassten 195 psychotherapieerfahrene Männer und Frauen. Eine Übersicht über die soziodemografischen und klinischen Merkmale der Stichprobe gibt Tabelle 1. Das durchschnittliche Alter beträgt 38,4 Jahre (SD $=11,8$; Range $=18-75) .74,9 \%$ der Teilnehmer sind Frauen; 96,1\% der Teilnehmer haben die deutsche Staatsangehörigkeit. Das Bildungsniveau in der Stichprobe ist hoch, wobei $30,6 \%$ über einen Hochschulabschluss, 29\% über Abitur und $32,1 \%$ über die mittlere Reife verfügen. Der überwiegende Teil der untersuchten Patienten ist berufstätig (72,9\%); der Anteil der Angestellten liegt bei 46,3\%; 17,2\% sind Schüler, Studierende oder Auszubildende. Diagnostisch handelt es sich vorwiegend um Patienten mit depressiven Störungen (62,1\%) und Angststörungen (52,8\%), wobei die Diagnose auf der Selbstaussage der Teilnehmer basiert.

Einen Überblick über die Charakteristika zurückliegender Behandlungen gibt Tabelle 2. 66,7\% der Teilnehmer hatten eine weibliche Therapeutin; ein Großteil war in ambulanter Behandlung (74,9\%). Die Mehrheit der Psychotherapien fand im Einzelsetting statt $(75,4 \%)$, umfasste in der Hälfte der Fälle zwischen 26-120 Therapiestunden (43,6\%) und lag im
Schnitt knapp 2 Jahre zurück (Range $=0$ 0-12,33 Jahre). 35 Personen (18\%) befanden sich zum Zeitpunkt der Befragung noch in psychotherapeutischer Behandlung. Über die Hälfte der Patienten berichteten von früheren Therapieerfahrungen $(63,1 \%)$, und ca. ein Fünftel hatte bereits eine frühere psychotherapeutische Behandlung vorzeitig abgebrochen (22,1\%). Individuelle Erwartungen an die Psychotherapie wurden im Schnitt eher erfüllt als nicht erfüllt $(\operatorname{Median}(\mathrm{M})=0,3, \mathrm{SD}=$ 2,3). Bei Betrachtung des Antwortverhaltens ergibt sich eine U-förmige Häufigkeitsverteilung: 37,4\% (n = 73) der Befragten gaben an, dass ihre Erwartungen an die Behandlung nicht erfüllt wurden (Werte von -1 bis -3$) ; 56,9 \%(n=111)$ gaben an, dass Erwartungen an die Psychotherapie erfüllt wurden. Die Beziehung zum Therapeuten wurde von den befragten Patienten als überwiegend positiv erlebt, und eine als negativ erlebte therapeutische Beziehung (Werte zwischen -1 und -3) wurde von $18 \%(n=35)$ berichtet.

\section{Häufigkeiten negativer Effekte und Itemkennwerte}

Tabelle 3 zeigt Itemkennwerte für negative Effekte von psychotherapeutischer Behandlung in den folgenden Lebensbereichen: intrapersonelle Veränderungen, Partnerschaft, Familie und Freunde, Arbeit, Stigmatisierung und therapeutisches Fehlverhalten. Dabei werden nur solche Effekte be- 


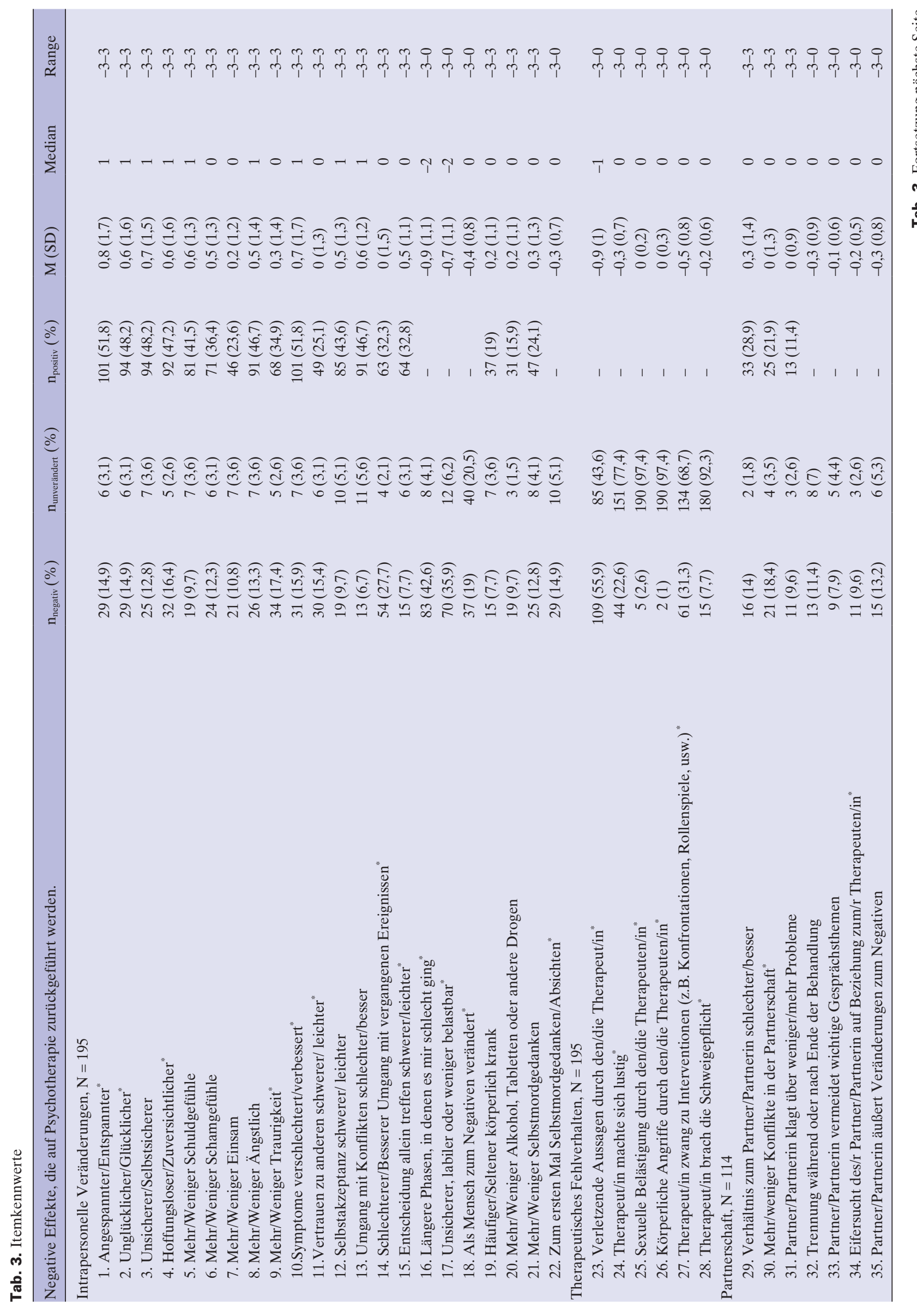




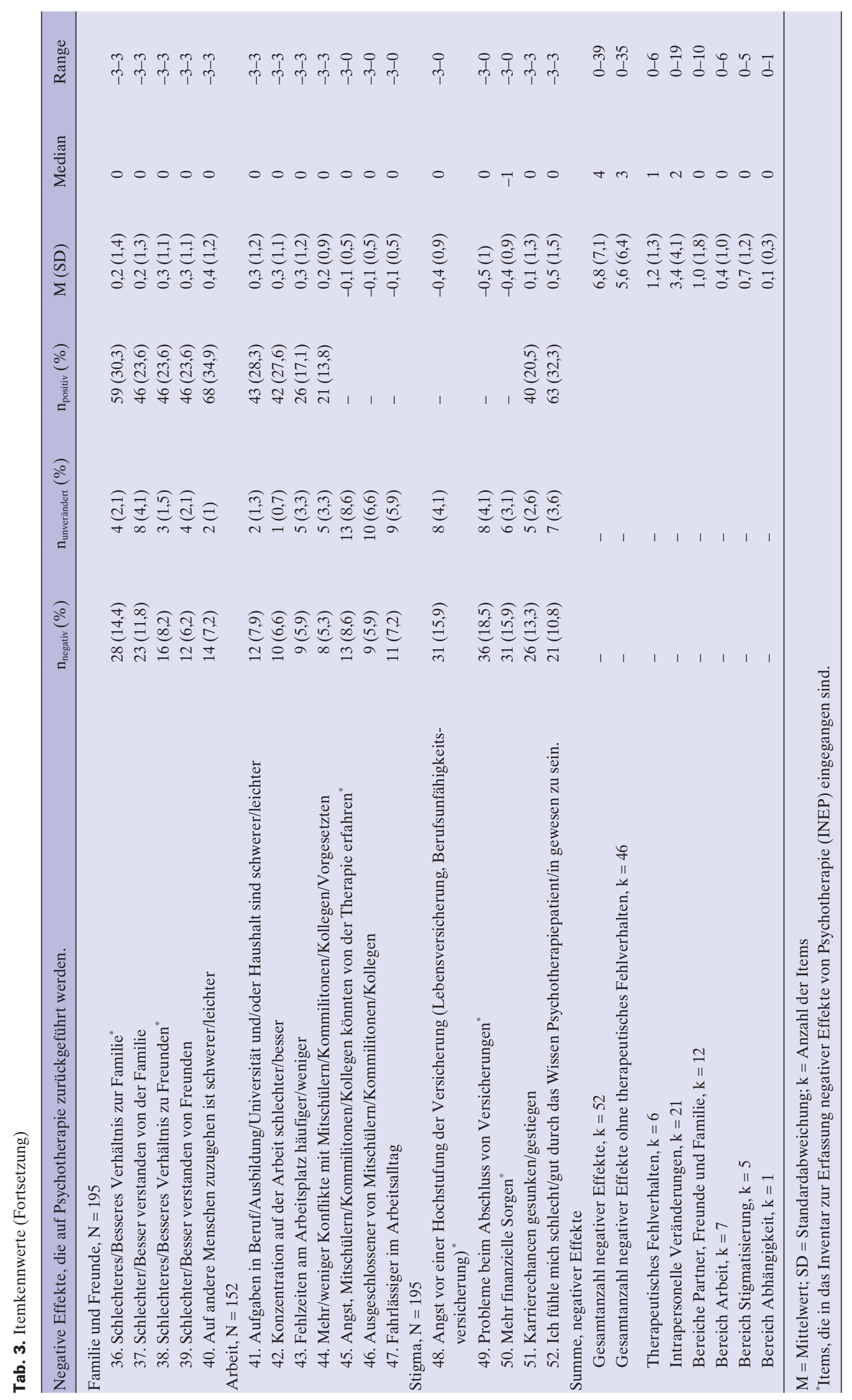


Tab. 4. Zusammenfassung der schrittweisen hierarchischen Regressionsanalyse zur Vorhersage der Anzahl berichteter negativer Effekte von Psychotherapie $(\mathrm{N}=$ 195)

\begin{tabular}{|c|c|c|c|}
\hline Variable & $\mathrm{B}$ & SE B & B \\
\hline 1. Schritt (Constant) & 5,885 & 0,427 & \\
\hline «Insgesamt wurden meine Erwartungen an die Therapie nicht erfüllt.» & $-1,185$ & 0,188 & $-0,41^{* *}$ \\
\hline 2. Schritt (Constant) & 5,122 & 0,481 & \\
\hline «Insgesamt wurden meine Erwartungen an die Therapie nicht erfüllt.» & $-1,180$ & 0,183 & $-0,41^{* * *}$ \\
\hline Setting: stationärer Klinikaufenthalt & 3,062 & 0,960 & $0,21^{* *}$ \\
\hline 3. Schritt (Constant) & 6,028 & 0,605 & \\
\hline «Insgesamt wurden meine Erwartungen an die Therapie nicht erfüllt.» & $-0,737$ & 0,257 & $-0,26^{* *}$ \\
\hline Setting: stationärer Klinikaufenthalt & 2,936 & 0,950 & $0,19^{* * *}$ \\
\hline «Ich erlebte die Beziehung zu meinem/r Therapeuten/in insgesamt als negativ.» & $-0,790$ & 0,326 & $-0,22^{*}$ \\
\hline 4. Schritt (Constant) & 4,920 & 0,774 & \\
\hline «Insgesamt wurden meine Erwartungen an die Therapie nicht erfüllt.» & $-0,672$ & 0,256 & $-0,24^{*}$ \\
\hline Setting: stationärer Klinikaufenthalt & 2,734 & 0,944 & $0,18^{* *}$ \\
\hline «Ich erlebte die Beziehung zu meinem/r Therapeuten/in insgesamt als negativ.» & $-0,846$ & 0,324 & $-0,23^{*}$ \\
\hline Frühere Therapieerfahrungen & 1,915 & 0,850 & $0,14^{*}$ \\
\hline
\end{tabular}

rücksichtigt, die die Teilnehmer ausdrücklich der psychotherapeutischen Behandlung zuschreiben. Wenn also ein Teilnehmer angibt, dass er sich nach der Therapie angespannter gefühlt habe, aber dies nicht direkt auf die Behandlung zurückführe, gilt dieses nicht als negativer Effekt der Psychotherapie. Da die Items im bipolaren Antwortformat formuliert sind, ergeben sich insgesamt 3 Kategorien von Effekten: negativ ( -3 bis -1 ), unverändert (0), und positiv (1 bis 3 ). Neben Häufigkeiten werden Mittelwert, Standardabweichung, Median und der Itemrange angegeben. Die interne Konsistenz des gesamten Fragenkatalogs $(\mathrm{k}=52)$ liegt bei einem Cronbachs $\alpha$ von 0,94 und ist damit als hoch einzuschätzen.

In der vorliegenden Stichprobe gaben 183 von 195 Befragten an, mindestens einen negativen Effekt nach oder während ihrer Psychotherapie erlebt zu haben. Die Häufigkeiten einzelner negativer Effekte lagen zwischen 1\% («Mein/e Therapeut/in hat mich körperlich angegriffen.») und 55,9\% («Ich fühlte mich durch die Aussagen meines/r Therapeuten/in verletzt»). Im Mittel gaben die Teilnehmer der Onlineumfrage an, 7 negative Effekte $(\mathrm{SD}=7,1$, Range $=0-39)$ erlebt $\mathrm{zu}$ haben, die sie auf ihre Behandlung zurückführen. Wenn man die Attribution außer Acht lässt, berichten die Teilnehmer ungefähr doppelt so viele negative Effekte $(\mathrm{M}=12,3, \mathrm{SD}=$ 7,9, Range $=0-39$ ).

Die Mittelwerte und Häufigkeiten der einzelnen Lebensbereiche sind in Tabelle 3 dargestellt. In dem Bereich «intrapersonelle Veränderungen» erlebten die Teilnehmer im Mittel die meisten negativen Effekte mit 15,8\% (Range $=6,7-$ 42,6\%; häufigstes Item: «Seit Abschluss meiner Therapie hatte ich längere Phasen, in denen es mir schlecht ging»). Am zweithäufigsten fanden sich erlebte oder befürchtete Stigmatisierungen durch andere, mit einer mittleren Häufigkeit von 14,9\% (Range $=10,8-18,5 \%$; häufigstes Item: «Seit Abschluss meiner Therapie habe ich Probleme, bestimmte Versicherungen abzuschließen»). Darauf folgten mit $12 \%$ negative Effekte im Bereich der Partnerschaft (Range $=7,9-18,4 \%$; häufigstes Item: «Seit Abschluss meiner Therapie erlebe ich in meiner Beziehung mehr Konflikte.») sowie mit 9,6\% negative Effekte in Familie und Freundeskreis (Range = 6,2-14,4\%; häufigstes Item: «Seit Abschluss meiner Therapie habe ich zu meiner Familie ein schlechteres Verhältnis»). Am wenigsten wurde im Mittel von negativen Veränderungen am Arbeitsplatz berichtet $(6,8 \%$; Range $=5,3-8,6 \%$; häufigstes Item: «Seit Abschluss meiner Therapie leide ich unter der Angst, meine Kollegen könnten von der Therapie erfahren»). 5 Teilnehmerinnen berichteten über sexuelle Belästigung durch ihre (jeweils weibliche) Therapeutin und 2 von ihnen über körperliche Angriffe durch die gleiche Therapeutin.

\section{Korrelate negativer Effekte von Psychotherapie}

Um mögliche korrelative Zusammenhänge negativer Effekte von psychotherapeutischer Behandlung zu untersuchen, wurde eine schrittweise (hierarchische) Regression durchgeführt. Als Outcome-Maß wurde dazu die absolute Anzahl an berichteten negativen Effekten $(\mathrm{M}=6,8 ; \mathrm{SD}=7,1$; Range = 0-39) verwendet (Summenwert, bereinigt um die Items zu therapeutischem Fehlverhalten, $\mathrm{k}=46$ ). Die Variablen, die in das Modell eingingen, wurden aufgrund einer bivariaten signifikanten Korrelation (Anhang 2; www.karger.com/?DOI=000367928) mit dem Outcome-Maß ausgewählt. Es ergaben sich signifikante korrelative Zusammenhänge zwischen der Anzahl negativer Effekte und einer als negativ beschriebenen therapeutischen Beziehung $\left(\mathrm{r}_{\text {Pearson }}=0,41, \mathrm{p}<0,01\right)$, unerfüllten Therapieerwartungen $\left(r_{\text {Pearson }}=0,43, p<0,01\right)$, dem Behandlungssetting («stationärer Klinikaufenthalt»; $\mathrm{r}_{\text {Spearman }}=0,27, \mathrm{p}<0,01$ ), früherer Therapieerfahrung $\left(\mathrm{r}_{\text {Pearson }}=0,18, \mathrm{p}<0,05\right)$, Therapieabbruch in der Vergangenheit ( Abbruch»; $\mathrm{r}_{\text {Spearman }}=0,17, \mathrm{p}<$ 
0,05), dem Berufsstatus («erwerbslos»; $\mathrm{r}_{\text {Spearman }}=0,27, \mathrm{p}<$ 0,01 ), Partnerschaftstatus ( «allein lebend»; $\mathrm{r}_{\text {Spearman }}=0,16, \mathrm{p}<$ $0,05)$ und der Diagnose «Persönlichkeitsstörung» $\left(\mathrm{r}_{\text {Spearman }}=\right.$ $0,14, p<0,05)-$ zu allen anderen Variablen fanden sich keine Zusammenhänge (z.B. Achse-1-Diagnosen, Alter, Therapieschule). Alle signifikanten Korrelate wurden dann in eine schrittweise hierarchische Regression eingeschlossen (Tab. 4). In Schritt 1 der hierarchischen Regression ist nur die Variable «Insgesamt wurden meine Erwartungen an die Therapie nicht erfüllt» enthalten. Den höchsten Erklärungszuwachs liefert dann die Variable «Behandlung im stationären Setting» (Schritt2; $\Delta \mathrm{R}^{2}=0,04 ; \mathrm{p}<0,01$ ). In den nächsten Schritten wurden die Variablen «negative therapeutische Beziehung» (Schritt 3; $\Delta \mathrm{R}^{2}=0,02 ; \mathrm{p}<0,01$ ) und «frühere Therapieerfahrungen» (Schritt $4 ; \Delta \mathrm{R}^{2}=0,02 ; \mathrm{p}<0,01$ ) aufgenommen. Insgesamt klärt das Modell über $20 \%$ der Varianz auf $\left(\Delta \mathrm{R}_{\text {korr }}^{2}=\right.$ $0,24 ; F(4,188)=16,4 ; p<0,01)$. Der Erklärungszuwachs ist für jeden Schritt signifikant. So wurden mehr negative Effekte angegeben, wenn der Teilnehmer die therapeutische Beziehung negativ bewertet hat, in einer stationären Behandlung war, frühere Therapieerfahrungen gemacht hatte und/oder seine Erwartungen an die durchgeführte Psychotherapie nicht erfüllt worden sind.

\section{Erstellung des Inventars zur Erfassung negativer Effekte von Psychotherapie (INEP)}

Bei der Konstruktion des Inventars wurde darauf geachtet, alle Lebens- und Funktionsbereiche des Patienten zu berücksichtigen. Mit der Faktorenanalyse soll untersucht werden, ob sich diese theoretischen Konstrukte auch statistisch in den Daten wiederfinden. Zunächst wurde eine Hauptkomponentenanalyse mit Varimax-Rotation über die 52 Items berechnet. Weitere Analysen ergaben mit Velicers MAP-Test eine 7-Faktorenstruktur und eine 5-Faktorenlösung mit der Parallelanalyse. Folgend wurden für beide Lösungen konfirmatorische Faktorenanalysen berechnet, wobei die 5-Faktorenstruktur $46,7 \%$ der Varianz aufklärte und die 7-Faktorenstruktur eine Varianzaufklärung von 55,8\% aufwies. Nach Untersuchung der einzelnen Faktorenstrukturen und Itemladungen sowie inhaltlichen Überlegungen erwies sich die 7-Faktorenstruktur als die zweckmäßigste. In Fällen von Doppelladungen wurden die Items nach inhaltlichen Überlegungen einem entsprechenden Faktor zugeordnet. Die Kennwerte der konfirmatorischen Analyse mit 7 Faktoren sind Tabelle 5 zu entnehmen.

Der erste Faktor (intrapersonale Veränderungen) umfasst 19 Items und beschreibt im weitesten Sinne negative Effekte im emotionalen Erleben und in der sozialen Funktionsfähigkeit. Der zweite Faktor (Partnerschaft) erfasst über 8 Items negative Effekte innerhalb der Partnerschaft, die durch die Therapie ausgelöst wurden. Faktor 3 (Stigmatisierung; 6 Items) beschreibt Befürchtungen, durch andere stigmatisiert zu werden und tatsächliche Benachteiligungen als Psychotherapiepatient zu erfahren. Die 4 Items in Faktor 4 (Emotionen) beschreiben negative Effekte, die einer depressiven
Symptomatik gleichen. Faktor 5 (Arbeitsplatz; 5 Items) und Faktor 7 (Familie und Freunde; 4 Items) beschreiben negative Effekte in den jeweiligen Lebensbereichen. Faktor 6 umfasst 6 Items und beschreibt therapeutisches Fehlverhalten durch den Behandler. Item 23 (Ich fühlte mich durch die Aussagen des Therapeuten/der Therapeutin verletzt) und Item 27 (Während der Therapie zwang mich mein Therapeut/meine Therapeutin Dinge zu tun, die ich eigentlich gar nicht wollte.) wurden aus inhaltlichen Gründen dem Faktor «therapeutisches Fehlverhalten» zugeordnet. Die Items innerhalb des Fragebogens verfügen über das gleiche Antwortformat mit je 2 unterschiedlichen Skalenniveaus und weisen eine hohe interne Konsistenz auf, was einen Teil der gemeinsamen Ladungen erklären kann.

Die Konstruktion des INEP erfolgte nach statistischen und inhaltlichen Kriterien, mit dem Ziel, ein ökonomisches Instrument zu erstellen. So gingen Items zur Erfassung therapeutischen Fehlverhaltens (Faktor 6) ungeachtet ihrer Häufigkeiten in den INEP ein, da diese Phänomene, wenn auch selten, als besonders schwerwiegend gelten und somit immer erfasst werden sollen. Im weiteren Verlauf wurden jeweils negative Effekte ausgewählt, die hohe Faktorladungen aufwiesen (Faktorladungen $>0,5$; Nebenladung $<0,35$ ) und/oder am häufigsten berichtet wurden (außerhalb des 1. Quartils, d.h. Häufigkeit $>16 \%$ ). Diese Kriterien wurden gewählt, um die relevanten Items pro Faktor zu identifizieren und seltene negative Effekte auszusortieren. Nach Umsetzung der inhaltlichen Überlegungen und Anwendung der statistischen Kriterien «Faktorladung» und «Häufigkeit» reduzierte sich die Anzahl der Items auf 26 (Tab. 5). In einem weiteren Schritt wurden diese Items auf mögliche Interkorrelationen überprüft, um Redundanzen zu vermeiden. Wenn diese Iteminterkorrelationen 0,60 überstiegen, wurden die Items jeweils inhaltlich zu einem neuen Item zusammengeschlossen. So wurden die Items 1, 2, 4, 9, 10 sowie Items 16, 17 und Items 48, 49 aufgrund hoher Iteminterkorrelationen ( $\mathrm{r}_{\text {pearson }}$ zwischen 0,65 und $0,74)$ zusammengefasst und haben nun folgenden Wortlaut:

- Items 1, 2, 4, 9, 10: «Seit Abschluss meiner Therapie fühle ich mich besser/schlechter.»

- Items 16, 17: «Seit Abschluss meiner Therapie hatte ich längere Phasen, in denen es mir schlecht ging.»

- Items 48, 49 «Seit Abschluss meiner Therapie habe ich Probleme mit Versicherungen (z.B. Lebensversicherung) bekommen bzw. Angst, dass Probleme entstehen könnten.»

Der Gesamtfragekatalog umfasste 20 Items. Zusätzlich wurde auf Anregung eines tiefenpsychologischen Experten ein neues Item generiert, das neben Item 15 die Abhängigkeit zum Therapeuten als einen negativen Effekt erfassen soll ( «Seit Abschluss meiner Therapie fühle ich mich von meinem Therapeuten/meiner Therapeutin abhängig.»). Die interne Konsistenz der Items, die in die Endversion eingingen $(\mathrm{k}=$ 21), lag bei einem Cronbachs $\alpha$ von 0,86 . Letztlich wurden 2 offene Fragen zu weiteren negativen Effekten und neben der Psychotherapie eine Spezifikation anderer Ursachen negati- 


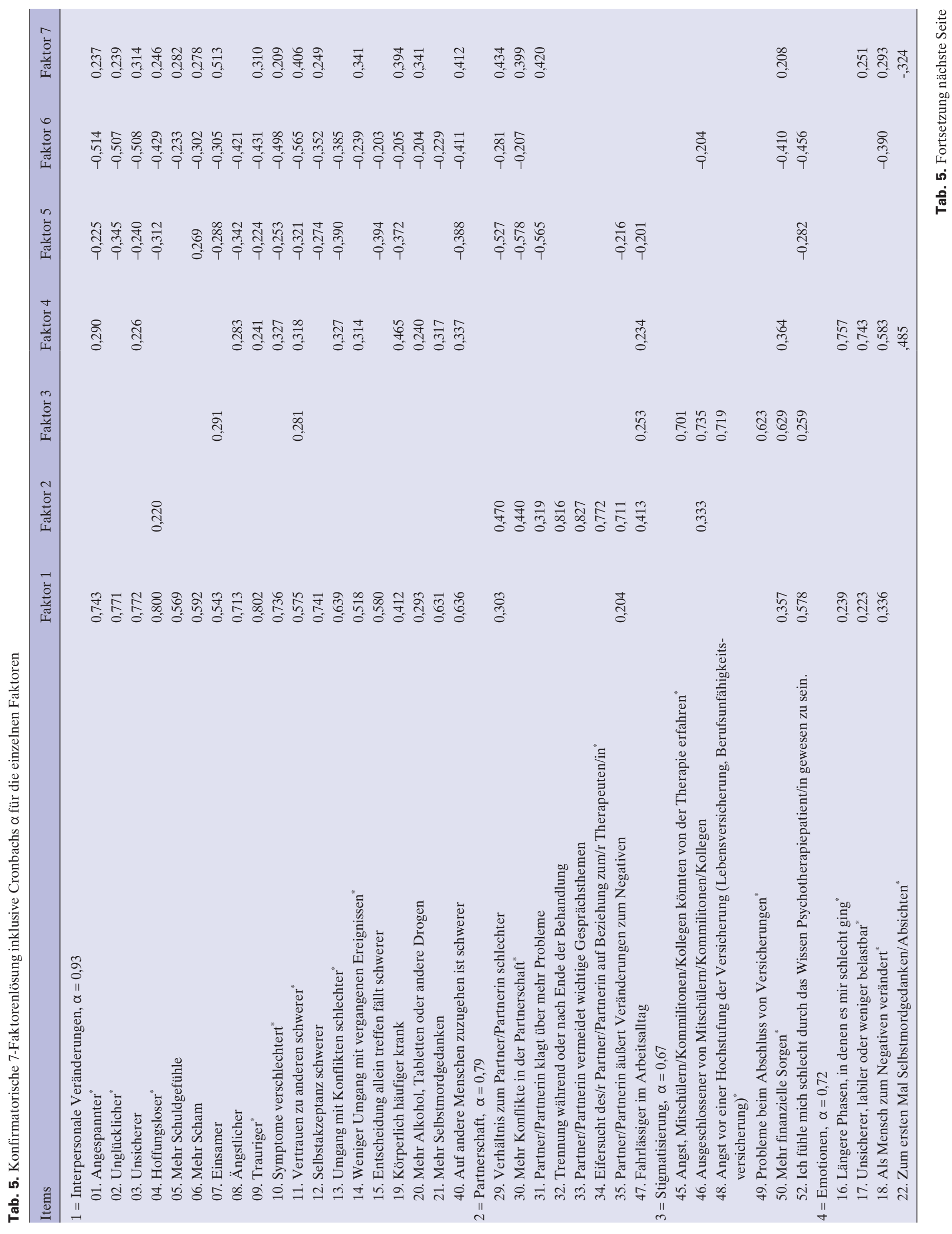




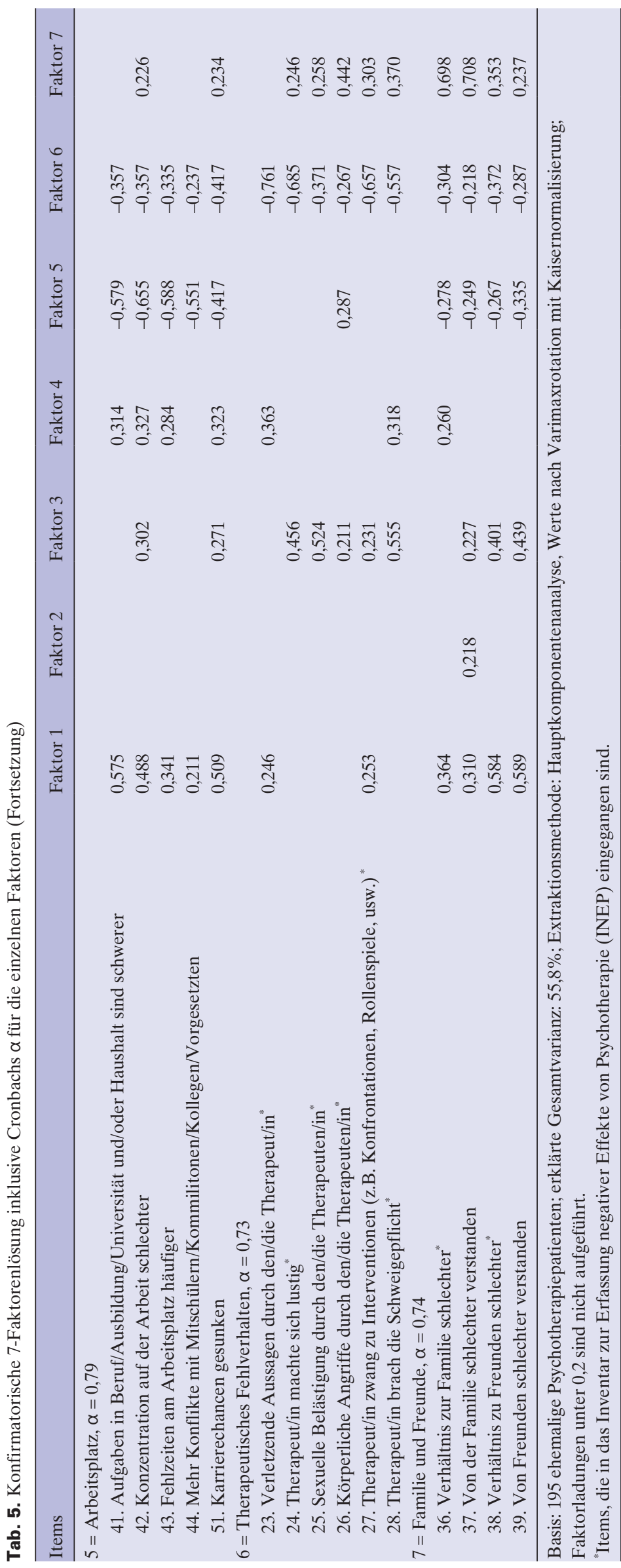

ver Effekte ergänzt, um Aufschluss über weitere negative Veränderungen zu bekommen und diese bei der Weiterentwicklung des Instrumentes berücksichtigen zu können. Das 7-stufige bipolare Antwortformat (4-stufig für unipolare Items) wurde beibehalten. Diese 21 Items (exklusive der 2 offenen Fragen) sind nun im INEP enthalten (Anhang 3; www.karger.com/? DOI=000367928).

\section{Diskussion}

Ziel dieser Erhebung war es, erste Hinweise zu Prävalenzen und Korrelaten für das Erleben negativer Effekte von Psychotherapie zu erhalten. Dazu wurde ein ökonomisches und reliables Selbstbeurteilungsverfahren entwickelt - das INEP mit 21 Items.

Grundsätzlich ist festzustellen, dass neben positiven auch eine nicht unerhebliche Anzahl negativer Effekte nach psychotherapeutischer Behandlung auftritt. Nur ein geringer Prozentsatz der Teilnehmer berichtete keinerlei negative Veränderungen durch ihre Behandlung. Die Prävalenzen einzelner negativer Effekte lagen zwischen 1 und $55,9 \%$, wobei die mittleren Häufigkeiten je nach Lebensbereich zwischen 6,8 und 15,8\% schwankten. Diese Prävalenzen liegen somit etwas höher als in vergleichbaren Studien $\mathrm{zu}$ therapeutischem Misserfolg [Lambert und Ogles, 2004]. Die ehemaligen Psychotherapiepatienten gaben dabei im Mittel am häufigsten negative Veränderungen in der eigenen Gedanken- und Gefühlswelt an (15,8\%) dort scheint das Risiko für das Erleben negativer Effekte besonders groß zu sein. Dies könnte in direktem Zusammenhang dazu stehen, dass ein Großteil der psychotherapeutischen Interventionen genau in diesem Bereich ansetzt und entsprechend auch dort den meisten Schaden anrichten kann. Anderseits könnten diese Häufigkeiten auch durch eine erhöhte Sensibilität in der Wahrnehmung von Veränderungen im eigenen Denken und Fühlen erklärt werden, die in der Psychotherapie erlernt wird.

Für den Bereich Partnerschaft stellten Hand und Lamontagne [1976] bei 33\% der Patienten vermehrte Partnerschaftskonflikte nach der Behandlung von Agoraphobie fest. In der hier beschriebenen Stichprobe fielen die Häufigkeiten negativer Effekte im Bereich der Partnerschaft mit $12 \%$ im Mittel geringer aus. Auf Einzelitems gaben 7,9-18,4\% der Befragten im Bereich Partnerschaft negative Effekte durch die Psychotherapie an. Betrachtet man nur die Patienten mit der Diagnose einer Angststörung $(\mathrm{n}=103)$, so gaben im Mittel 7,8\% der Befragten negative Effekte im Bereich Partnerschaft an; etwas mehr berichten Depressionspatienten (8,5\%). Patienten mit Persönlichkeitsstörungen beschrieben im Mittel weniger negative Effekte (6,3\%) innerhalb der Partnerschaft. Dabei muss jedoch die Validität der selbstberichteten Diagnosen berücksichtigt werden. 
Neben diesen Effekten beschrieben Patienten auch gravierende und besorgniserregende Veränderungen. So gab eine substanzielle Anzahl von Teilnehmern (14,9\%) an, im Rahmen der Behandlung erstmals Suizidgedanken erlebt zu haben. Daneben berichteten $1-2,6 \%$ der Teilnehmer von sexueller Belästigung und körperlichen Übergriffen durch den Behandler - therapeutisches Fehlverhalten, das zu weiteren negativen Veränderungen für die Patienten führen kann. Diese Zahlen fallen teilweise geringer aus als in bisherigen Studien. So berichtet Tschan [2005], dass es bei etwa $10 \%$ der Psychotherapien zu sexuellen Kontakten zwischen Therapeut und Patient kommt. Diese Befunde bestärken die Relevanz des Themas in ethischer und auch rechtlicher Hinsicht und machen es unabdingbar, sich mit negativen Effekten psychotherapeutischer Behandlung auseinanderzusetzen.

Das neu entwickelte Inventar hat sich in der vorliegenden Erhebung als objektiv und reliabel erwiesen. Faktorenanalytisch konnte die theoretisch erstellte Einteilung der negativen Effekte in verschiedene Lebens- und Funktionsbereiche in großen Teilen bestätigt und so für die Erstellung eines ökonomischen Instruments (INEP) genutzt werden. Folgestudien müssen die gefundene Faktorenstruktur auf ihre Replizierbarkeit überprüfen.

In Hinblick auf die Validität des INEP können bisher noch keine Aussagen getroffen werden. Innerhalb der hier durchgeführten Regressionsanalyse werden erste Hinweise zu möglichen Korrelaten negativer Effekte von Psychotherapie gegeben, wobei die Richtung der Zusammenhänge noch ungeklärt ist. Risikofaktoren für negative Effekte scheinen eine schlechte therapeutische Beziehung und unerfüllte Therapieerwartungen aufseiten des Patienten zu sein. Außerdem treten negative Effekte häufiger im stationären klinischen Umfeld auf als in ambulanten Behandlungssituationen. Ein Grund dafür könnte im erhöhten Symptomschweregrad der stationären Patienten liegen, der die Wahrnehmung eines Therapieeffekts als negativ möglicherweise begünstigt. In dieser Studie lagen die nötigen Daten zur Beurteilung der Symptomschwere der Teilnehmer allerdings nicht vor. Zusätzlich werden innerhalb stationärer Behandlungen in der Regel nur wenige Einzelsitzungen durchgeführt, und nicht selten finden zusätzlich Therapeutenwechsel statt, wodurch der Aufbau einer vertrauensvollen Beziehung zwischen Patient und Therapeut erschwert wird. Neben diesen therapiespezifischen Merkmalen kam mit der Diagnose der Persönlichkeitsstörung ein dezidiertes Patientenmerkmal zum Vorschein, das mit einem erhöhten Erleben von negativen Effekten einhergeht. Es ist anzunehmen, dass dieses Ergebnis eng mit den fehlenden interpersonellen Fähigkeiten dieser Patientengruppe zusammenhängt und somit im therapeutischen Kontext Probleme entstehen können, die die Gefahr für subjektiv erlebte negative Effekte erhöhen. Die Gründe für die oben genannten Zusammenhänge sind gewiss vielfältig, können an dieser Stelle jedoch nur spekulativ erörtert werden.
Keine Zusammenhänge konnten zwischen der Gesamtanzahl an negativen Effekten und den soziodemografischen Patientenmerkmalen oder spezifischen Therapieverfahren gefunden werden. Jedoch zeigten sich auf Itemebene Unterschiede bezüglich der Therapieschulen: So berichteten Patienten einer Verhaltenstherapie (VT) häufiger, von ihrem Therapeuten $\mathrm{zu}$ Interventionen gezwungen worden $\mathrm{zu}$ sein $(36,5 \%=)$. Demgegenüber stand der Vergleich von Patienten einer psychodynamischen Therapie (PD; 23,0\%), einer Gesprächstherapie (GT; 30,0\%) und sonstigen Therapieformen $(40,0 \%)$. Patienten einer GT gaben in der vorliegenden Stichprobe längere Phasen der Niedergeschlagenheit nach der Therapie an (GT $=50 \%$; Sonstige $=50,0 \%$; VT $=40,5 \%$ PD $=37,7 \%$;), während Pateinten einer PD sich häufiger durch die Aussagen ihres Therapeuten verletzt fühlten (PD = $65,6 \% ; \mathrm{GT}=60 \%$; VT $=50 \%$; Sonstige $=40,0 \%$ ).

Diese Variablen sind erste Hinweise zu konvergenten und divergenten Konstrukten und müssen in Folgeerhebungen weiter untersucht werden. Eine spannende Frage für Nachfolgestudien wäre hierzu, ob die negative Effekte z.B. auch nach erfolgreichen Therapien oder vermehrt nach fehlgeschlagener Behandlung auftreten.

\section{Limitationen und zukünftige Forschung}

Auch wenn gezeigt werden konnte, dass negative Effekte infolge einer psychotherapeutischen Behandlung auftreten und mithilfe des INEP erfasst werden können, ist die vorliegende Studie noch in einigen Punkten begrenzt. So handelte es sich bei den Studienteilnehmern ausschließlich um Psychotherapieerfahrene, die über das Internet rekrutiert wurden. Die Ergebnisse können daher nicht ohne Weiteres verallgemeinert werden und sollten in weiteren Patientenstichproben überprüft werden. Auch kann nicht ausgeschlossen werden, dass bei der vorliegenden Stichprobe ein Selbstselektionsbias vorlag. Aus anschließenden qualitativen Interviews mit einigen Probanden $(n=35)$ wissen wir jedoch, dass sowohl Patienten teilnahmen, die sich sehr positiv über ihre Behandlung äußerten, als auch Therapieverdrossene. In Hinblick auf die Repräsentativität der Stichprobe verglichen Gosling und Kollegen [2004] mehrere Internetstichproben mit traditionellen Paper-and-Pencil-Stichproben und kamen zu dem Schluss, dass die Validität der erhobenen Daten in beiden Studien identisch war.

Im Rahmen der neu entwickelten Begriffsdefinition werden die untersuchten Effekte als negativ beschrieben, wobei die Valenz der einzelnen Effekte nicht direkt im Fragebogen abgefragt wird. Wir haben versucht, dieser Valenz indirekt über das bipolare Itemformat näherzukommen. Zusätzlich kann bei den erfragten Effekten davon ausgegangen werden, dass sie zunächst einen Stressor für den Patienten darstellen und demnach erst einmal als negativ empfunden werden. So bringt die Trennung vom Partner, auch wenn diese erwünscht ist, einen hohen Disstressfaktor mit sich. Folglich kann dabei jedoch nicht ausgeschlossen werden, dass ein negativer Effekt 
für den einzelnen Patienten zu einem späteren Zeitpunkt auch positive Aspekte haben kann. Dies ist jedoch nicht Gegenstand des Fragebogens und der dargestellten Untersuchung.

Als weitere Einschränkung gilt, dass mit dem INEP nur die Perspektive der Klienten erfasst wird und damit die externe Validität hinsichtlich der Behandlerperspektive eingeschränkt ist. Obwohl die subjektive Erfahrung des Patienten von zentraler Bedeutung ist und auf diese bei der Erforschung von negativen Effekten nicht verzichtet werden darf, bietet das Instrument nicht die Möglichkeit, die Übereinstimmung der erlebten negativen Effekte mit den von Therapeuten beobachteten Effekten zu prüfen. Haupt und Linden [2011] haben hierzu ein Instrument entwickelt, das unerwartete Effekte aus Therapeutenperspektive erfasst und eine sinnvolle Ergänzung zum INEP darstellen könnte.

Zusätzlich muss die Validität mancher Patientenangaben im Rahmen der Befragung als unsicher bewertet werden (z.B. zu Diagnosen, Therapieverfahren usw.). So ist die gesamte Befragung aus retrospektiver Sicht durchgeführt worden, wobei unter den Teilnehmern unterschiedlich viel Zeit seit der Beendigung der Therapie vergangen war und die Antworten damit von unterschiedlicher Qualität sein könnten.

\section{Implikationen}

Bisher herrscht vorrangig die Meinung, dass Psychotherapie im «schlimmsten Fall» gar nicht wirkt, also im Gegensatz $\mathrm{zu}$ anderen, vor allem medizinischen Interventionen, nur wenige bis gar keine negativen Effekte hat und, wenn richtig durchgeführt, den Patienten nicht schaden kann. Jedoch zeigt die vorliegende Untersuchung, dass Psychotherapie, neben allen positiven Effekten, auch mit unerwünschten negativen Effekten einhergeht. Den Behandlern sollte bewusst sein, dass Psychotherapie einen Einfluss über die Hauptsymptoma- tik hinaus auch auf andere Lebens- und Funktionsbereiche hat. Sowohl Klienten als auch Therapeuten und Kandidaten in Ausbildung sollten über die Möglichkeit von negativen Effekten aufgeklärt und dafür sensibilisiert werden. Dies könnte, ähnlich wie bei der Erfassung von Erfolgskriterien, zusätzlich über eine standardmäßige Evaluierung negativer Effekte erfolgen. Selbst wenn manche (aber bei Weitem nicht alle) negativen Effekte als Teil des therapeutischen Prozesses gesehen werden können und vielleicht sogar notwendig sind, so gilt es trotzdem, deren Häufigkeit zu reduzieren. Im Kontext der gesetzlichen Verpflichtung (Patientenrechtegesetz) bei der Richtlinien-Psychotherapie, Patienten vor Beginn einer Behandlung über mögliche negative Effekte zu informieren, liefert der INEP zudem eine wertvolle Informationsquelle. Neben diesen klinischen Implikationen muss die systematische Untersuchung negativer Effekte in unterschiedlichen Settings und aus unterschiedlichen Perspektiven weitergeführt werden. Nur dann können wir besser verstehen, wie und unter welchen Umständen welche negativen Effekte von Patienten berichtet werden und wie wir zukünftig damit umgehen wollen.

\section{Dank}

Unser Dank geht an Prof. Dr. Franz Caspar, Prof. Dr. Thomas Fydrich, Prof. Dr. Harald Gründel, Prof. Dr. Peter Henningsen, Prof. Dr. Bernd Löwe, Prof. Dr. Jürgen Margraf, Prof. Dr. Bernhard Strauss und Prof. Dr. Dietmar Schulte für die wertvolle Unterstützung und das hilfreiche Feedback zum Konstruktionsprozess des INEP.

\section{Disclosure Statement}

Die Autoren erklären, dass keine Interessenkonflikte vorliegen.

\section{Literatur}

Bortz J, Schuster C: Statistik für Human- und Sozialwissenschaftler, ed 7. Heidelberg, Springer, 2010.

Gosling SD, Vazire S, Srivastava S, John OP: Should we trust web-based studies? A comparative analysis of six preconceptions about internet questionnaires. Am Psychol 2004;59:93-104.

Hand I, Lamontagne Y: The exacerbation of interpersonal problems after rapid phobia-removal. Psychother 1976;13:405-411.

Haupt M, Linden M: Nebenwirkungen und Nebenwirkungserfassung in der Psychotherapie - Das ECRS-ATR-Schema. Psychoth Soz 2011;13:9-27.

Heuer H, Rösler F, Tack WH: Merkmale und Gütekriterien psychologischer Tests; in Krohne HW Hock M (eds): Psychologische Diagnostik: Grundlagen und Anwendungsfelder. Stuttgart, Kohlhammer, 2007, pp 23-83.
Lambert MJ, Ogles BM: The efficacy and effectiveness of psychotherapy; in Lambert MJ (ed): Bergin and Garfield's Handbook of Psychotherapy and Behavior Change, ed 5. New York, Wiley and Sons, 2004, pp 139-193.

Mohr D: Negative outcome in psychotherapy: a critical review. Clin Psychol - Sci Pr 1995;2:1-27.

Nestoriuc Y, Rief W: Risiken und Nebenwirkungen von Verhaltenstherapie; in Linden M, Strauß B (eds): Risiken und Nebenwirkungen von Psychotherapie. Berlin, MWV, 2012, pp 59-74.

Schmoll D: Nebenwirkungen bei psychoanalytisch orientierten Therapien. Psychotherapeut 2012;57: 395-401.
Smith ML, Glass G: Meta-analysis of psychotherapy outcome studies. Am Psychol 1977;32:752-760.

Tschan W: Missbrauchtes Vertrauen. Sexuelle Grenzverletzung in professionellen Beziehungen. Basel, Karger, 2005.

Wampold B: The Great Psychotherapy Debate: Models, Methods, and Findings. Mahwah, Taylor and Francis, 2001

Westermann S, Kesting M-L, Lincoln T: Being deluded after being excluded? How emotion regulation deficits in paranoia-prone individuals affect state paranoia during experimentally induced social stress. Behav Ther 2012;43:329-340. 